\title{
Anticancer effects of lactoferrin: underlying mechanisms and future trends in cancer therapy
}

\author{
Yunlei Zhang, Cristovao F Lima, and Ligia R Rodrigues
}

Lactoferrin has been widely studied over the last 70 years, and its role in diverse biological functions is now well known and generally accepted by the scientific community. Usually, alterations of the lactoferrin gene in cells are associated with an increased incidence of cancer. Several studies suggest that exogenous treatment with lactoferrin and its derivatives can efficiently inhibit the growth of tumors and reduce susceptibility to cancer. None of these studies, however, reported a consistent outcome with regard to the mechanisms underlying the anticancer effects of lactoferrin. In this review, the association of lactoferrin with cancer is thoroughly discussed, from lactoferrin gene expression to the potential use of lactoferrin in cancer therapy. Lactoferrin cytotoxicity against several cancers is reported to occur in distinct ways under different conditions, namely by cell membrane disruption, apoptosis induction, cell cycle arrest, and cell immunoreaction. Based on these mechanisms, new strategies to improve the anticancer effects of the lactoferrin protein and/or its derivatives are proposed. The potential for lactoferrin in the field of cancer research (including as a chemotherapeutic agent in cancer therapy) is also discussed.

(C) 2014 International Life Sciences Institute

\section{INTRODUCTION}

Lactoferrin, an $80-\mathrm{kDa}$ protein with iron-binding ability, was first discovered in mammary secretions but is synthesized by most mammalian tissues. ${ }^{1}$ It is present in high concentrations in human (1.0 to $\approx 3.2 \mathrm{mg} / \mathrm{mL}$ ) and bovine milk $(0.02$ to $\approx 0.35 \mathrm{mg} / \mathrm{mL})$ and in lower concentrations in a variety of secretions derived from epithelial cells as well as in the second granules of neutrophils. ${ }^{2,3}$ This wide distribution indicates that lactoferrin is possibly involved in multiple important physiological activities.

Many relevant biological functions have been reported for lactoferrin, including anticancer, antibacterial, antiviral, antifungal, anti-inflammatory, and immune regulatory activities. ${ }^{4,5}$ Some studies indicate that the iron-binding ability of lactoferrin and the interaction between lactoferrin and its specific receptors are responsible for the diverse biological functions demonstrated thus far. ${ }^{4}$ In addition, lactoferrin-derived peptides and the iron-saturated form of lactoferrin (hololactoferrin) have also been shown to be efficient anticancer and antibacterial drugs. ${ }^{2}$ The most-studied lactoferrin-derived peptide is bovine lactoferricin $\mathrm{B}$, a cationic antimicrobial peptide derived from the N-terminal of the protein lactoferrin. ${ }^{6}$ This peptide, like the protein, has been the object of increasing interest. However, the detailed mechanisms underlying the broad range of functions of lactoferrin and its derivatives still need to be clarified. Insight into the role of lactoferrin in cancer development and progression has been provided through published data on the anticancer mechanisms of lactoferrin. For example, studies have shown that the downregulation or silencing of

Affiliations: $Y$ Zhang and $L R$ Rodrigues are with the CEB - Centre of Biological Engineering, University of Minho, Braga, Portugal. $Y$ Zhang is with the College of Life Science, Hunan Provincial Key Laboratory of Microbial Molecular Biology - State Key Laboratory Breeding Base of Microbial Molecular Biology, Hunan Normal University, Changsha, China. CF Lima is with the CITAB - Centre for the Research and Technology of Agro-Environmental and Biological Sciences, Department of Biology, University of Minho, Braga, Portugal.

Correspondence: LR Rodrigues, CEB - Centre of Biological Engineering, University of Minho, Campus de Gualtar, 4710-057 Braga, Portugal. E-mail: Irmr@deb.uminho.pt. Phone: +351-253-604-401. Fax: +351-253-604-429.

Key words: cancer therapy, cytotoxicity, gene, lactoferrin, mechanisms, protein 
lactoferrin or cytosolic lactoferrin (delta lactoferrin) genes in cells leads to an increase of malignant tumors. ${ }^{7,8}$ In contrast, the proliferation of cancer cells is blocked following restoration of the lactoferrin gene. ${ }^{9,10}$ Additionally, several reports have shown that lactoferrin and/or its derivatives can inhibit the growth of tumors both in vitro and in vivo. ${ }^{11-19}$

Besides having nutritional value, lactoferrin and/or its derivatives may have considerable potential in the prevention and treatment of cancer. The present review aims to provide an outline of their mechanisms of action against cancer as well as their possible applications in the field of cancer research.

\section{LACTOFERRIN GENE EXPRESSION AND DECREASED CANCER SUSCEPTIBILITY}

Lactoferrin protein is widely distributed in mammalian cells. The synthesis of lactoferrin mRNA is regulated either directly or indirectly by estrogen, retinoic acid, mitogen, and growth factors. The multiple response elements lie mainly in the 3 short, but complex, modules within $400 \mathrm{bp}$ of the transcription site of the lactoferrin gene. ${ }^{20}$ For example, the human lactoferrin gene promoter (P1) contains 2 chicken ovalbumin upstream promoter transcription factor elements, one of which is overlapped with the estrogen response element, while the other is present within a composite response element adjacent to the estrogen response element. ${ }^{21}$ Overexpression of the chicken ovalbumin upstream promoter transcription factor can block the estrogenstimulated responses. ${ }^{22}$ The infection-responsive promoter in lactoferrin gene has also been characterized. Lipopolysaccharide-responsive regions of the promoter contain 1 signal transducer and activator of transcriptions (STAT3) site, 3 activator protein 1 sites, and 8 nuclear factor- $\mathrm{\kappa B}$ sites. Lactoferrin promoter may respond to infections via the nuclear factor- $\kappa B$ pathway. ${ }^{23}$ This observation explains the large increase in lactoferrin concentrations in mammary secretions infected by bacteria. The expression of the lactoferrin gene is stimulated mainly by bacterial lipopolysaccharide and double-strand RNA. ${ }^{24}$ In addition, expression of lactoferrin in mammary epithelial cells is mediated by changes in cell shape and the actin cytoskeleton. ${ }^{25}$ Furthermore, miR-214, expressed in 23 species, was found to be directly involved in lactoferrin expression and the lactoferrin-mediated cancer susceptibility in mammary epithelial cells. ${ }^{26}$

Some of these response elements in the lactoferrin gene, such as the estrogen and infection response elements, may be implicated in an increased cancer incidence. Estrogen can promote DNA synthesis and cell proliferation by turning on estrogen-responsive genes. Exposure to an excess of estrogen will greatly increase the incidence of breast cancer. ${ }^{27,28}$ On the other hand, it is well known that 1 out of 6 cancers develops from infection, ${ }^{29}$ and suppression of infection plays an important role in cancer therapy after chemotherapy or bone marrow transplantation..$^{30}$ The regulatory elements responding to pathogenic factors are involved in expression of the lactoferrin gene. This indicates that lactoferrin probably acts as a negative regulator in the human body to repress the acute reaction to estrogen or to kill virus and bacterial infection by sharply raising the protein expression. Additionally, potent antimicrobial and antiviral effects have been associated with the use of exogenous lactoferrin., These properties can be very helpful in the prevention of infections and cancer recurrence after chemotherapy treatments. Complete knowledge about these lactoferrin gene regulatory factors constitutes a useful basis for designing novel strategies for possible uses that may include the exogenous supply of the protein lactoferrin.

\section{LACTOFERRIN GENE EXPRESSION AND GENETIC VARIATION IN CANCER CELLS}

A relationship between lactoferrin gene expression and susceptibility to breast cancer was first proposed by Furmansiki et al., ${ }^{31}$ who found that lactoferrin-associated RNase activity was present at lower levels in milk obtained from a consanguineous community with a high incidence of breast cancer in India. Recent studies also showed that both the $\mathrm{P} 1$ and $\mathrm{P} 2$ promoter regions of the lactoferrin gene are downregulated or silenced in several cancer cell lines. ${ }^{7}$ This downregulation is much more apparent for delta lactoferrin, the expression of which is either significantly diminished (in BT-20 and MCF-7 cells) or practically absent (in MDA-MB-231, T-47D, and HBL 100 cells) in cancerous tissue, ${ }^{8}$ though it is regularly expressed in normal tissues. Moreover, stable expression of delta lactoferrin or overexpression of lactoferrin led to significant inhibition of cancer cell growth as well as to a weakened potential to form in vivo tumors after orthotopic transplantation. ${ }^{9,10}$

Besides revealing the distinct levels of lactoferrin expression in normal as well as in cancerous tissues, the evaluation of the lactoferrin gene structure showed that the degree and pattern of methylation are notably altered in malignant breast cells. ${ }^{32,33}$ For instance, a lactoferrin cDNA clone extracted from human breast tissue was used to evaluate changes in the restriction fragment length and the methylation patterns of DNA from normal peripheral blood, from leukemia cells from patients, and from leukemia and breast cancer cell lines. Patterns of DNA methylation in malignant cells were found to be highly variable. However, DNA was generally less methylated than in normal cells. ${ }^{33}$ In addition, methylation assays conducted in a model of nasopharyngeal cancer demon- 
strated that methylation also occurred in the promoter of the lactoferrin gene, which is linked to decreased lactoferrin gene expression. Simultaneously, abnormal migration patterns of the lactoferrin gene were also found in cancer cells. More specifically, the alternation generally takes place in the lactoferrin exons $4,5,13,14$, and $15 .{ }^{34}$

Genetic variation of the lactoferrin gene significantly increases cancer susceptibility. The changes in methylation of the promoter or first exon may have the same effect as mutations of various tumor suppressor genes or proto-oncogenes. Carcinogenesis can result from aberrations in genomic DNA methylation, including hypermethylation and hypomethylation of the promoter or first exon of cancer-related genes. ${ }^{35}$ Recent studies showed that, apparently, cancer cells have a relatively high occurrence of genetic polymorphisms, gene mutations, and promoter hypermethylation in the lactoferrin gene. ${ }^{32-34}$ All these observations suggest a close relationship between alterations of the lactoferrin gene and an increasing incidence of carcinomas. Hence, the use of lactoferrin as a novel cancer-specific marker may have applications in diagnosing cancer as well as in gauging the prognosis of cancer patients at different stages. Alternatively, lactoferrin may have uses as a therapeutic agent in chemoprevention or in clinical practice. Since the restoration of lactoferrin expression successfully depresses the growth of tumors, the lactoferrin gene could be further explored as a new target site for cancer gene therapy.

\section{CHEMOPREVENTIVE POTENTIAL OF ORAL LACTOFERRIN}

The World Health Organization reported that, in 2012, 14.1 million people were newly diagnosed with cancer, 8.2 million cancer patients died, and 32.6 million people were living with cancer (within a 5-year diagnosis) worldwide. $^{36}$ This means the incidence of cancer is still very high and that, currently, there is no effective cure, especially in patients at advanced stages of the disease. Therefore, preventing the development of carcinomas is crucial for decreasing the currently high mortality of cancer. Some epidemiological studies have shown that more than two-thirds of cancers could be prevented through the adoption of an appropriate lifestyle. ${ }^{37} \mathrm{~A}$ diet rich in preventive agents is one of the strategies proposed to decrease the incidence of cancer and other diseases. ${ }^{38}$ Milk-derived lactoferrin is considered a functional protein and reaches a level of $7 \mathrm{~g} / \mathrm{L}$ in colostrum. ${ }^{3}$ The multifunctional lactoferrin could, thus, play a role in cancer prevention.

Indeed, a large number of reports provide clear evidence that oral administration of bovine lactoferrin can effectively decrease the development of chemically induced cancers in animal models. ${ }^{39-42}$ Bovine lactoferrin is a relatively stable protein that can be active even after passing through the gastrointestinal tract as partially degraded fragments (derivative peptides). ${ }^{43}$ These fragments, with a molecular mass over $20 \mathrm{kDa}$, contain the receptor-binding regions of the protein as well as the anticancer active regions. ${ }^{43}$ An example of these fragments is the lactoferricin B peptide. ${ }^{17,18}$ Moreover, these fragments can be internalized by specific lactoferrin receptors in the intestinal brush border membrane. ${ }^{44}$ The oral administration of $0.2 \%$ or $2 \%$ bovine lactoferrin was found to decrease by $32.5 \%$ and $42.5 \%$, respectively, the occurrence of colon carcinogenesis in azoxymethane-treated F344 rats. ${ }^{39}$ In a study conducted with the 7, 12-dimethylbenz(a)anthracene-induced hamster buccal pouch model, the incidence of carcinogenesis was reduced by a diet containing $0.2 \%$ bovine lactoferrin. ${ }^{41}$ Moreover, oral administration of bovine lactoferrin showed chemopreventive activity against tongue, ${ }^{39}$ esophagus, and lung carcinogenesis in rats. ${ }^{42}$ The suggested chemopreventive effects could be due to the multiple functions of lactoferrin, which include stimulation of the immune response ${ }^{39,40}$ modulation of the carcinogen-metabolizing enzymes and the oxidant-antioxidant profile in the target organs, ${ }^{41}$ and inhibition of angiogenesis. ${ }^{40}$ Regulation of the immune function by lactoferrin may be a key factor in the mechanisms of action involved in cancer prevention. Presently, the extraction of bovine lactoferrin from milk and whey is an industrial reality. Therefore, it is plausible that, in the future, lactoferrin-containing supplements or the consumption of lactoferrin-enriched dietary products might be recommended to prevent or delay the onset of cancer.

\section{ANTICANCER ACTIVITY AND MECHANISMS OF ACTION}

Although the exact mechanisms involved in the anticancer activity of lactoferrin are still unclear, they can be generally grouped into extracellular effects, intracellular effects, and immunostimulation. The extracellular effects are related mainly to the interaction of lactoferrin with the cell membrane and membrane receptors, ${ }^{45,46}$ while the intracellular effects suggested by most studies are related mostly to cell apoptosis and cell cycle arrest (Table 1). ${ }^{9,11-15,17,19,47-52}$ The immunostimulatory action of lactoferrin is achieved primarily by activating immune cells to release tumor cytotoxic effectors. ${ }^{49,53-56}$ The proposed mechanisms underlying the cytotoxicity of lactoferrin or its derivatives against cancer cells are diverse and are derived from in vitro experiments (Figure 1). Moreover, findings from in vivo experiments are generally similar. ${ }^{39-42,49}$ 


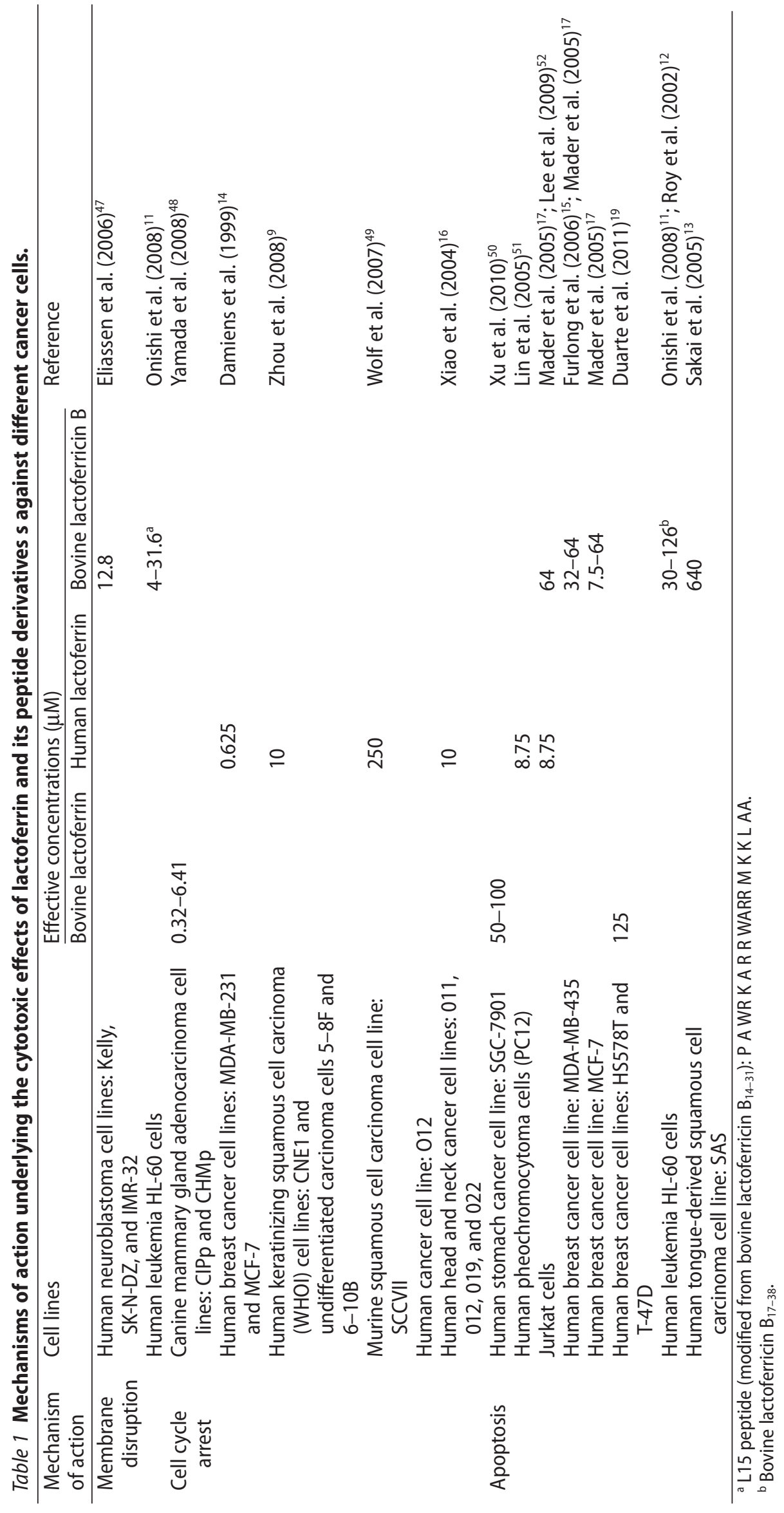




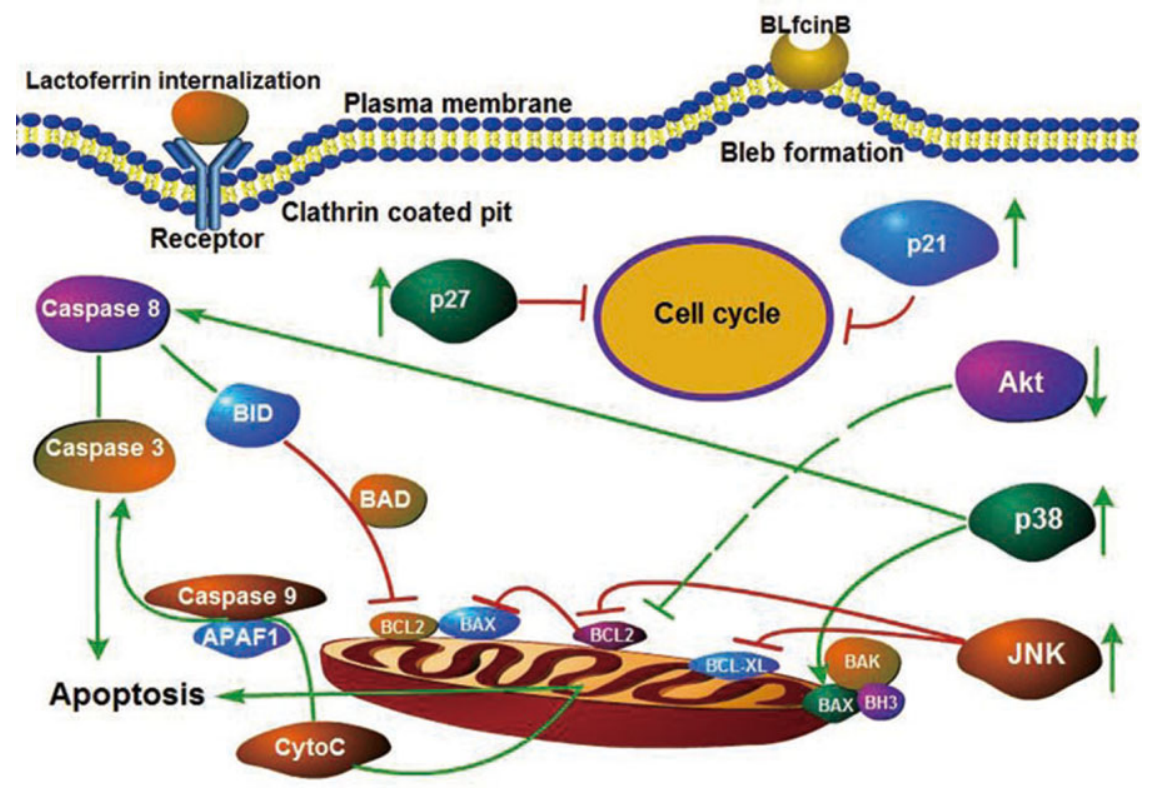

Figure 1 Multiple signaling pathways potentially underlying the cytotoxic effects of lactoferrin and its derivatives against cancer cells. This figure provides further explanation of the mechanisms of action of lactoferrin and its derived peptides against cancer cells shown in Table 1. Bovine lactoferrin and human lactoferrin exert cytotoxic effects against cells by inducing cell cycle arrest and apoptosis, while bovine lactoferricin B inhibits cell growth by triggering mitochondrial-related apoptosis and disrupting the cell membrane. All three inhibit the activity of Akt (the down arrow) but activate p21, p27, p38, and JNK (the up arrow) and induce the release of caspase-8, caspase-3, and cytochrome $\mathrm{C}$.

Abbreviations: BLfcin B, bovine lactoferricin B; CytoC, cytochrome C; JNK, C-Jun N-terminal kinase; Akt, protein kinase B; APAF1, apoptotic protease activating factor 1 .

\section{Toxic effects of lactoferrin and its derived peptides on the cell membrane}

Lactoferrin and its derived peptides were found to be easily internalized by cells..$^{57,58}$ In Jurkat human lymphoblastic T-cells, lactoferrin enters cells via receptormediated endocytosis and is almost completely degraded in the lysosomal compartment. ${ }^{58}$ The lactoferrin-active peptide lactoferricin B also exhibits conformationdependent uptake efficiency. ${ }^{59}$ Increased membrane permeability may produce subtle changes in the membrane's barrier function that promotes cell death. At low concentrations, lactoferrin can increase cytolysis, while at high concentrations it modulates cytolysis, depending on the target cell phenotype. ${ }^{60}$ Many studies demonstrated the presence of lactoferrin receptors on the tumor cell surface. Heparan sulfate plays an important role in the regulation of the binding of lactoferrin and lactoferricin $B$ with the receptors. ${ }^{57-59,61}$ Indeed, when it is removed, a reduction in membrane binding and cellular uptake of lactoferrin and lactoferricin B occurs. ${ }^{59,61}$ However, the characterization of these receptors is still under investigation, since different cell types appear to express their own specific lactoferrin receptors. ${ }^{46}$

Lactoferricin B is proposed to exert a higher potential activity against tumor cells than lactoferrin because it is a cationic antimicrobial peptide. ${ }^{45}$ Some cationic antimicrobial peptides have shown a selective activity against cancer cells, thus constituting a promising group of novel anticancer agents with a new mode of action and a broad spectrum of anticancer activity. ${ }^{45,59}$ Bovine lactoferricin B is composed of 25 amino acid residues (the residues 17-41 of bovine lactoferrin) that contain a disulfide bridge between two Cys residues in positions 19 and 36. ${ }^{6,62}$ This disulfide bond may not be essential for maintaining the antibacterial activity of bovine lactoferricin B, but it is crucial for maintaining the activity against tumor cells. ${ }^{62,63}$ In aqueous solution, bovine lactoferricin B loses the $\alpha$-helical portion of the parent protein, adopting a slightly twisted antiparallel $\beta$-sheet amphipathic structure, with nearly all of the hydrophobic residues lining up on one face of the peptide. ${ }^{6}$ This change causes it to bear similarities with several major classes of cationic antimicrobial peptides, which contain disulfide bridges and antiparallel $\beta$-strands and form amphipathic helices upon binding to membranes. Moreover, lactoferricin B exists as a $\alpha$-helix structure when it is still linked to the parent lactoferrin, and this is also an important feature of membrane lytic peptides. ${ }^{64}$ When cationic antimicrobial peptides come into contact with the cell membrane, they undergo conformational changes that enable their association with the membrane; consequently, the cell lyses. ${ }^{64}$ 
It is important to notice that this mechanism is still not entirely understood. Moreover, some cationic antimicrobial peptides can trigger apoptosis by disrupting the mitochondrial membranes. ${ }^{65} \quad$ Fluorescence-labeled lactoferricin B has been found in the mitochondrial membrane of neuroblastoma cells. ${ }^{47}$ Additionally, many studies suggest that the membrane may constitute one of the targets through which lactoferrin and lactoferricin B exert their anticancer effects. ${ }^{11,47}$

In addition to their ability to disrupt the cell membrane, cationic antimicrobial peptides could also contribute to the selective toxicity of lactoferricin (peptide derived from lactoferrin) against cancer cells. Several studies have demonstrated that lactoferricin B or its derivatives selectively kill cancer cells without adversely affecting normal cells. ${ }^{17,18,47}$ This could be due to the subtle differences that exist between the cell membranes of normal cells and those of cancer cells. The most pronounced difference is a much more negatively charged cell membrane in cancer cells. ${ }^{66,67}$ Indeed, the relatively high levels of negatively charged molecules are likely to be more attractive to the cationic antimicrobial peptides, such as lactoferricin B or its variants. Therefore, these peptides are more prone to bind to cancer cell membranes, leading to cell death, than to normal cells.

\section{Cell apoptosis}

Cell apoptosis induced by lactoferrin and lactoferricin B has been described as the pivotal pathway by which these peptides exert their cytotoxic effects against various cancer cells. However, the apoptosis pathway that they trigger, the extrinsic (Fas receptor family) or the intrinsic (mitochondria-associated) pathway, apparently varies, depending on the cell type used. ${ }^{9,11,17,47,50-52,68}$

Akt acts as an antiapoptotic signaling molecule and plays a critical role in the function of cancer cells. ${ }^{69}$ For example, when stomach cancer cells SGC-7901 were treated with lactoferrin, the phosphorylated Akt and several key proteins involved in the signaling pathway were decreased, but expression of phosphorylated glycogen synthase kinase-3 $\beta$ Ser256 and phosphorylated caspase-9 Ser 196 were increased, which suggests that the lactoferrin-induced apoptosis of stomach cancer cells SGC-7901 may be regulated via the Akt pathway. ${ }^{50}$ Furthermore, lactoferrin was also found to induce a stressrelated mitogen-activated protein kinase pathway in Jurkat $\mathrm{T}$ cells, where the c-Jun N-terminal kinase (JNK) associated with $\mathrm{Bcl}-2$ was presumed to be the pathway responsible for the lactoferrin-induced apoptosis. ${ }^{52}$ Lactoferrin treatment induced the activation of caspase- 9 and caspase-3 and increased the level of $\mathrm{Bcl}-2$ phosphorylation. When JNK activation was abolished, lactoferrin-treated Jurkat cells did not undergo cell death..$^{52}$ Additionally, lactoferrin induced apoptosis in the colon mucosa of azoxymethane-treated rats by enhancing Fas expression. ${ }^{68}$ After oral administration of lactoferrin, Fas protein expression was augmented more than 2.5fold, and the active forms of both caspase- 8 and caspase- 3 were also higher. Immunohistochemical analysis revealed the presence of Fas-positive and apoptotic cells preferentially at the site of lactoferrin-mediated tumor inhibition. ${ }^{68}$ In addition, lactoferrin was found to protect PC12 cells from FasL-induced apoptosis at a low concentration $(50 \mu \mathrm{g} / \mathrm{mL})$, while it triggered apoptosis through inhibition of the expression of phosphorylated extracellularsignal-regulated kinase $1 / 2$ at a high concentration $(700 \mu \mathrm{g} / \mathrm{mL}) .^{51}$

Most studies with lactoferricin B demonstrate that cells undergo the mitochondrial-related apoptosis pathway. ${ }^{11,17,47}$ The cationic antimicrobial peptide structure of lactoferricin B may facilitate its interaction with both surface death receptors and intracellular apoptosisrelated proteins. An example includes the apoptosis induction in oral squamous carcinoma cells by JNK/ SAPK activation using lactoferricin B. In the process, the JNK pathway directly activates the mitochondrial death machinery. ${ }^{70}$

\section{Cell cycle arrest}

Besides apoptosis, cell cycle arrest is another crucial pathway involved in the cytotoxic effects of lactoferrin against cancer cells. Lactoferrin could induce cell growth arrest by decreasing phospho-Akt to increase the expression and activity of p21 ${ }^{\mathrm{Cip} 1}$ and $\mathrm{p} 27^{\mathrm{Kip} 1} \cdot{ }^{14,16}$ After exposure to lactoferrin, the MDA-MB-231 cells stopped at the G1 to $S$ transition phase of the cell cycle, which was associated with a dramatic decrease in the levels of proteins Cdk2, cyclin E, and Cdk4, accompanied by an augmented expression of the Cdk inhibitor p2 $1^{\text {Cip1 }}{ }^{14}$ Cell cycle arrest induced by lactoferrin was also demonstrated using nasopharyngeal carcinoma cells. The expression of cyclin D1 and phosphorylation of retinoblastoma protein was downregulated, while expression of p21 and p27 was enhanced. ${ }^{9}$ Similar cell cycle arrest profiles were found in murine squamous cell carcinoma SCCVII, human cancer cell line O12, and canine mammary gland adenocarcinoma cell line after treatment with lactoferrin. ${ }^{48,49}$

\section{Immunostimulation and iron regulation}

Immunostimulation is assumed to be a key factor for the in vivo anticancer effects of lactoferrin. Both innate and adaptive immunity are involved in the immunoreaction induced by lactoferrin or its derivatives (Figure 2) ${ }^{49,53-56}$ As a result of the cyclic change in 


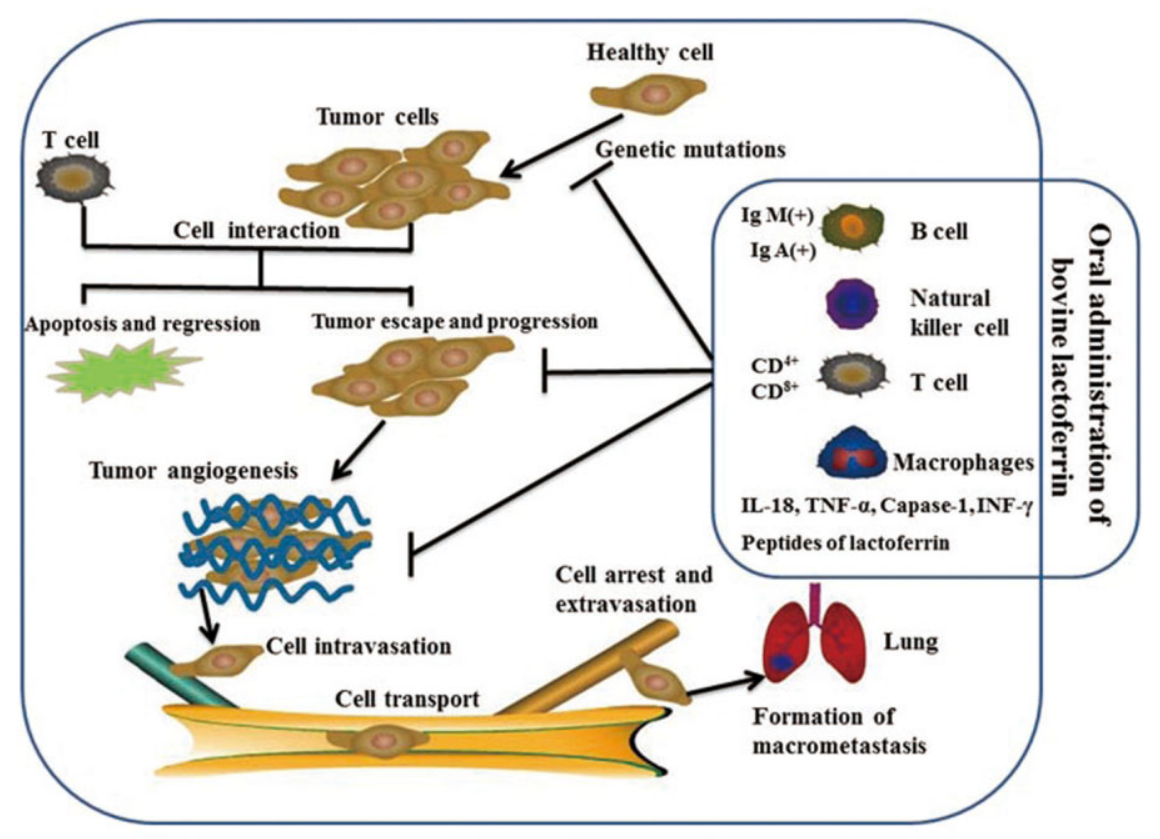

Figure 2 In vivo anticancer effects of bovine lactoferrin. Oral administration of bovine lactoferrin efficiently activates $B$ and $T$ cells and increases the effect of natural killer cells and macrophages, while the expression of IFN- $\gamma$, TNF- $\alpha$, caspase- 1 , and IL-18 increases significantly. A cascade effect with these proteins further amplifies the anticancer effect, which efficiently inhibits carcinogenesis, tumor proliferation, and tumor angiogenesis during tumor development.

lactoferrin concentration, the secretory components, such as immunoglobulin (Ig) A and IgG, increase sharply at proestrus in the uterus and decline in the other stages. ${ }^{71}$ In cancer, lactoferrin functions predominantly by activating a strong Th1 response and the release of anticancer killer cells. ${ }^{53}$ The recruitment of lymphocytes, mainly including $\mathrm{CD}^{+}$and $\mathrm{CD}^{+}$, reaches up to a 20 -fold increase within treated animals after oral administration of bovine lactoferrin. ${ }^{49}$ The tumor-infiltrating lymphocytes can greatly inhibit the proliferation of cancers. ${ }^{72}$

The expression of interferon- $\gamma$, tumor necrosis factor- $\alpha$ (TNF- $\alpha$ ), caspase-1, and interleukin (IL)-18, as well as $\operatorname{IgM}^{+}$and $\operatorname{IgA}^{+} \mathrm{B}$ cells, is also significantly increased in the small intestine after bovine lactoferrin treatment. ${ }^{54-56,73,74}$ Actually, a cascade effect exists among these proteins. Caspase- 1 is known to cleave pro-IL-18 to generate mature IL-18. ${ }^{54}$ IL-18 is an interferon- $\gamma$ inducing cytokine. ${ }^{75}$ Further, IL-18 has an important role in the expression of TNF- $\gamma$ in T cells and natural killer cells and as a stimulator of TNF- $\alpha$ and several other cytokines. IL-18 can also enhance Th1 and natural killer cell responses and generate $\mathrm{CD} 8^{+}$effector T cells. ${ }^{76}$ Systemic and intralesional administration of IL-18 significantly inhibited the growth of tumors. ${ }^{75}$ In addition, lactoferrin can increase the effector functions of natural killer cells and macrophages at low concentrations and enhance the production of nitric oxide that has been reported to sensitize tumors to chemotherapy. ${ }^{60,77}$ More- over, orally administered bovine lactoferrin was found to systemically restrict $\mathrm{VEGF}_{165}$-mediated angiogenesis in rats. ${ }^{40,78}$ The combined effects of these factors may, therefore, lead to the eradication of tumors and blocking of pathogens. In addition, lactoferrin can flexibly bind and release several kinds of metal-related cations, such as $\mathrm{Zn},{ }^{2+} \mathrm{Fe},{ }^{3+} \mathrm{Cu}^{2+} \mathrm{Mn}^{3+}$, and $\mathrm{Ga}^{3+}{ }^{3+}$ Indeed, lactoferrin acts as one of the main iron regulators in the human body and is responsible for maintaining systemic iron homeostasis. ${ }^{79}$ Iron balance is necessary for cell growth and enzyme activity. If unbalanced, iron can become a potentially toxic element, leading to the formation of free radicals. These radicals induce a cellular redox imbalance by producing oxidative stress, which may be related to oncogenic stimulation. ${ }^{80}$ As such, lactoferrin may be an effective chelator to maintain the balance of iron in vivo. Furthermore, iron chelators have been shown to possess antiproliferative activity both in vitro and in vivo. ${ }^{81}$

\section{ENHANCING THE ANTICANCER EFFECTS OF LACTOFERRIN AND ITS DERIVATIVES}

Currently, it is recognized and has been demonstrated that lactoferrin and its derivatives possess anticancer activities. ${ }^{16-18,48}$ Moreover, their use in combination with other agents has proven highly successful. ${ }^{15,82-87}$ Therefore, it is thought that lactoferrin and its derivatives 
synergize with other known anticancer agents or delivery systems in order to generate their cytotoxic effects against cancer.

The ability to specifically target a tumor is one of the desired properties of an ideal antitumor drug. An appropriate delivery system can be very helpful in getting lactoferrin and its derivatives to the tumor. Massodi et al. ${ }^{82}$ developed an elastin-like polypeptide carrier to deliver the peptide derivative of bovine lactoferrin-L12. ${ }^{18}$ The thermally responsive assemblage is soluble in aqueous solutions at $37^{\circ} \mathrm{C}$ but aggregates near $41^{\circ} \mathrm{C}$, which makes it an ideal carrier for targeting solid tumors when treatment is focused on hyperthermia, for example during radiotherapy. On the other hand, liposomes are an efficient drug delivery system and can greatly enhance the therapeutic potential of the encapsulated compounds. Liposomeentrapped iron-free lactoferrin (apolactoferrin) demonstrated a clearly enhanced inhibitory effect on the growth of B16-F10 cells. ${ }^{83}$ In addition, a brain-targeted chemotherapeutical delivery system, doxorubicin-loaded lactoferrin-modified procationic liposome, efficiently raised the uptake and cytotoxicity of lactoferrin against glioma C6 cells. ${ }^{87}$ Furthermore, several studies successfully used adenovirus to target lactoferrin to cancers in animal models. ${ }^{85,86}$ These recombinant adenoviruses could significantly suppress the growth of tumors by releasing lactoferrin within solid tumors.

Combining lactoferrin or its variants with recognized anticancer agents is another effective way to increase the anticancer activity of chemotherapeutic agents. Tumors in mice fed hololactoferrin were completely eliminated with a single injection of known chemotherapeutic agents. ${ }^{77}$ In vitro experiments in breast cancer cells showed bovine lactoferricin to have greatly augmented cytotoxic effects when combined with C6-ceramide and tamoxifen. ${ }^{15}$ Another interesting study revealed that a combination of human neutrophil peptide- 1 and human lactoferrin could kill cancer cells without affecting normal cells. Neither human neutrophil peptide-1 nor human lactoferrin could demonstrate this effect individually. ${ }^{84}$ The selective cytotoxicity against cancers is the most important feature of any antitumor drug. Therefore, these case studies suggest that the combination of lactoferrin or its derivatives with other anticancer agents could represent an efficient way to boost the cytotoxic effects and, consequently, the performance of these agents against cancers, with limited side effects.

\section{CLINICAL APPLICATIONS AND FUTURE TRENDS FOR LACTOFERRIN IN CANCER THERAPY}

Although there is still some uncertainty about the mechanisms underlying the cytotoxicity of lactoferrin and its derivatives against cancer, there is no doubt about its potential anticancer activity. Since lactoferrin and its derivatives are food-derived components, and thus nontoxic, they constitute an interesting alternative to chemoprevention and the currently used anticancer drugs. In addition, its stability through the gastrointestinal tract is beneficial if oral administration is envisaged. All of these features are believed to be essential for an efficient drug. To date, several clinical trials have been initiated to evaluate the therapeutic effects of lactoferrin in cancer therapy, such as for non-small-cell lung cancer (clinical trial registration no. NCT00706862) and renal cell carcinoma (clinical trial registration no. NCT00095186). Since the trial for non-small-cell lung cancer was ongoing at the time of manuscript preparation, a comprehensive evaluation of the results was not available. Lactoferrin has been found to be useful for reducing some clinical complications associated with chemotherapy, such as anemia, ${ }^{88}$ and immunoreaction stimulated by lactoferrin is thought to be a key factor in cancer therapy.

Most of the data on the anticancer effects of lactoferrin are based on in vitro studies, in which lactoferrin and its derivatives have been shown to greatly inhibit the proliferation of various cancer cells. ${ }^{11-17,19,47,50-52}$ This means that their cytotoxicity is probably due to the direct contact between lactoferrin and cancer cells. Thus, the direct and indirect actions of lactoferrin should be fully explored so that a greater anticancer effect can be obtained in vivo (Figure 3). The direct action of lactoferrin can be achieved by intratumoral or intravenous injection. Unfortunately, though, lactoferrin can be quickly cleared after intravenous administration. ${ }^{89}$ In light of this, perhaps a tumor-targeting delivery system, which will prevent the protein from degrading before it reaches the tumor cells, would improve the anticancer effects in vivo. Currently, several carrier vectors, involving systemic targeting as well as intracellular targeting, are available to deliver anticancer agents. Both the widely used liposomes and the emergent nanoparticles have proved to be efficient carriers whose further use in clinical practice is supported. ${ }^{90}$ They may be modified with specific ligands to target tumor cells or to just remain in circulation longer, thus providing a slow release of lactoferrin or its variants, thereby persistently activating the immune system. This approach has the potential to greatly reduce any possible adverse effects and increase the agent's performance against cancer. In addition, the combination of lactoferrin and its derivatives with established anticancer agents has been shown to result in increased killing of cancer cells in vitro ${ }^{82,83,87}$ and should be further explored. With this approach, the first clinical use of lactoferrin is envisaged as an adjuvant therapeutic agent in cancer patients. This will not only take advantage of the immunotherapeutic effects of lactoferrin, it will 


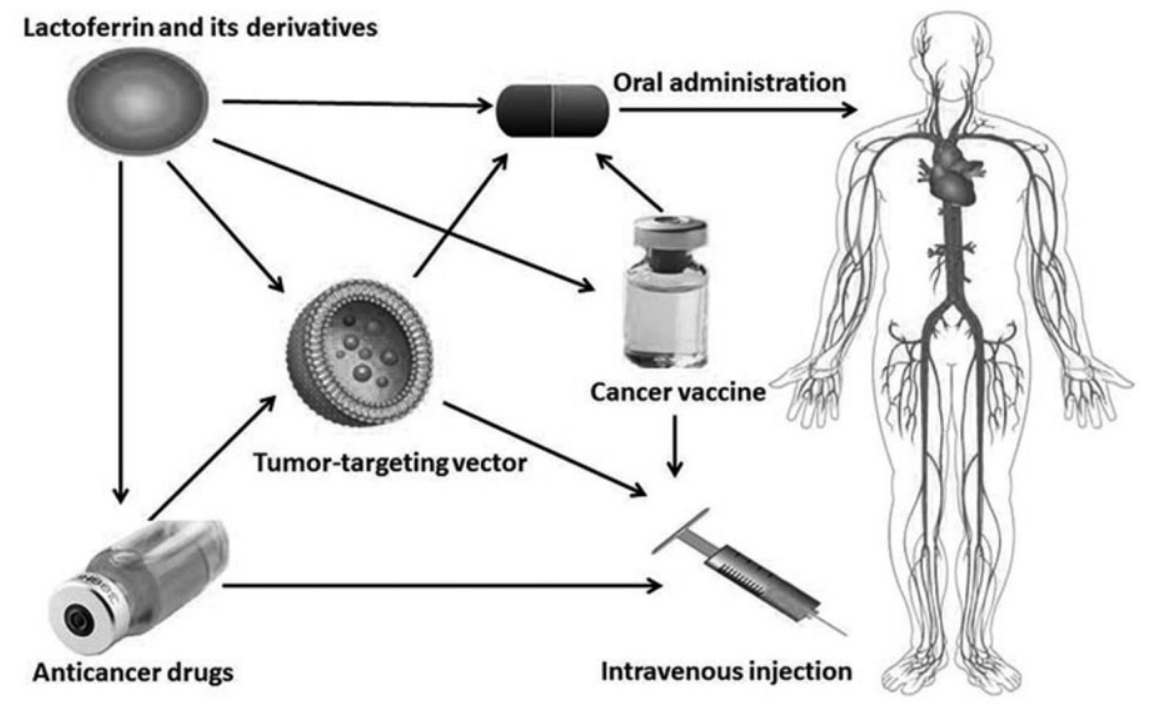

Figure 3 Potential modes of application of lactoferrin and its derivatives for prevention and/or therapy of cancer.

also provide anticancer effects superior to those obtained when chemotherapy agents are used alone.

\section{CONCLUSION}

Lactoferrin is a multifunctional protein that can be widely found in most mammalian cells. Its expression is regulated by several factors to maintain homeostasis. Silencing or downregulation of the lactoferrin gene in cells is usually related to the occurrence of certain diseases, especially carcinomas. Restoration of lactoferrin gene expression can effectively inhibit the proliferation of cancer cells. The response elements of disease-related factors in the lactoferrin promoter and its adjacent regions could partly explain the vital role this protein plays in the prevention of serious diseases. Cytotoxic assays also demonstrated the inhibitory effects of lactoferrin and its derivatives against the proliferation of multiple cancers, thus suggesting a potential role in cancer prevention. However, the mechanisms involved in the cytotoxicity of lactoferrin and its derivatives against cancer are derived from in vitro experiments and are not always conclusive. ${ }^{11,12,16,17,19}$ Fortunately, however, the conclusions drawn from in vivo experiments about the mechanisms involved are similar, i.e., tumors are eliminated due to the lactoferrin-stimulated increase in tumor-killer cells. ${ }^{49,54-56}$

Although clinical trials involving the use of lactoferrin in cancer therapy are ongoing, there are still no reports about the use of lactoferrin in clinical practice. The relatively low cytotoxicity of lactoferrin and its derivatives as compared with known anticancer drugs, along with the lack of data about the mechanisms of action, is likely hampering the clinical use of lactoferrin in cancer treatment. However, as previously mentioned, lactoferrin and its derivatives could be an effective anticancer treatment modality if combined with other therapeutic agents or if encapsulated in carriers after appropriate modifications. It is interesting to note that some studies have already begun to use these approaches to increase the cytotoxic effects of lactoferrin and its derivatives. ${ }^{15,82,83}$ As combination therapy becomes increasingly popular, it is likely that lactoferrin will continue to be studied for its potential value as a primary or adjuvant agent in the treatment of cancer.

\section{Acknowledgments}

Funding. Financial support was received from the Erasmus Mundus External Cooperation Window (Y), the Strategic Project PEst-OE/EQB/LA0023/2013, and the Fundação para a Ciência e a Tecnologia (project reference RECI/BBB-EBI/0179/2012; project no. FCOMP-010124-FEDER-027462).

Declaration of interest. The authors have no relevant interests to declare.

\section{REFERENCES}

1. Farnaud S, Evans RW. Lactoferrin - a multifunctional protein with antimicrobial properties. Mol Immunol. 2003;40:395-405.

2. Rodrigues $\mathrm{L}$, Teixeira J, Schmitt F, et al. Lactoferrin and cancer disease prevention. Crit Rev Food Sci Nutr. 2009;49:203-217.

3. Masson PL, Heremans JF. Lactoferrin in milk from different species. Comp Biochem Physiol B. 1971;39:119-129.

4. Adlerova L, Bartoskova A, Faldyna M. Lactoferrin: a review. Veterinarni Medicina. 2008:53:457-468.

5. Gibson RJ, Bowen JM. Biomarkers of regimen-related mucosal injury. Cancer Treat Rev. 2011;37:487-493. 
6. Zhou N, Tieleman DP, Vogel HJ. Molecular dynamics simulations of bovine lactoferricin: turning a helix into a sheet. Biometals. 2004;17:217-223.

7. Hoedt $E$, Hardivillé $S$, Mariller $C$, et al. Discrimination and evaluation of lactoferrin and delta-lactoferrin gene expression levels in cancer cells and under inflammatory stimuli using TaqMan real-time PCR. Biometals. 2010;23:441-452.

8. Benaïssa M, Peyrat JP, Hornez L, et al. Expression and prognostic value of lactoferrin mRNA isoforms in human breast cancer. Int J Cancer. 2005;114:299306

9. Zhou Y, Zeng Z, Zhang W, et al. Lactotransferrin: a candidate tumor suppressordeficient expression in human nasopharyngeal carcinoma and inhibition of NPC cell proliferation by modulating the mitogen-activated protein kinase pathway. Int J Cancer. 2008;123:2065-2072.

10. Breton M, Mariller C, Benaïssa $\mathrm{M}$, et al. Expression of delta-lactoferrin induces cell cycle arrest. Biometals. 2004;17:325-329.

11. Onishi J, Roy MK, Juneja LR, et al. A lactoferrin-derived peptide with cationic residues concentrated in a region of its helical structure induces necrotic cell death in a leukemic cell line (HL-60). J Pept Sci. 2008;14:1032-1038.

12. Roy MK, Kuwabara $\mathrm{Y}$, Hara $\mathrm{K}$, et al. Peptides from the $\mathrm{N}$-terminal end of bovine lactoferrin induce apoptosis in human leukemic (HL-60) cells. J Dairy Sci. 2002;85:2065-2074.

13. Sakai $T$, Banno $Y$, Kato $Y$, et al. Pepsin-digested bovine lactoferrin induces apoptotic cell death with JNK/SAPK activation in oral cancer cells. J Pharmacol Sci. 2005;98:41-48.

14. Damiens E, El Yazidi I, Mazurier J, et al. Lactoferrin inhibits G1 cyclin-dependent kinases during growth arrest of human breast carcinoma cells. J Cell Biochem. 1999;74:486-498.

15. Furlong SJ, Mader JS, Hoskin DW. Lactoferricin-induced apoptosis in estrogennonresponsive MDA-MB-435 breast cancer cells is enhanced by C6 ceramide or tamoxifen. Oncol Rep. 2006;15:1385-1390.

16. Xiao $\mathrm{Y}$, Monitto $\mathrm{CL}$, Minhas $\mathrm{KM}$, et al. Lactoferrin down-regulates $\mathrm{G} 1$ cyclindependent kinases during growth arrest of head and neck cancer cells. Clin Cancer Res. 2004;10:8683-8686.

17. Mader JS, Salsman J, Conrad DM, et al. Bovine lactoferricin selectively induces apoptosis in human leukemia and carcinoma cell lines. Mol Cancer Ther 2005;4:612-624.

18. Yang N, Strøm MB, Mekonnen SM, et al. The effects of shortening lactoferrin derived peptides against tumour cells, bacteria and normal human cells. J Pept Sci. 2004; 10:37-46.

19. Duarte DC, Nicolau A, Teixeira JA, et al. The effect of bovine milk lactoferrin on human breast cancer cell lines. J Dairy Sci. 2011;94:66-76.

20. Teng CT, Gladwell W, Beard C, et al. Lactoferrin gene expression is estrogen responsive in human and rhesus monkey endometrium. Mol Hum Reprod. 2002;8:58-67.

21. Teng $C$. Mouse lactoferrin gene: a marker for estrogen and epidermal growth factor. Environ Health Perspect. 1995;103:17-20.

22. Liu $\mathrm{Y}$, Yang N, Teng CT. COUP-TF acts as a competitive repressor for estrogen receptor-mediated activation of the mouse lactoferrin gene. Mol Cell Biol. 1993;13:1836-1846

23. Zheng J, Ather JL, Sonstegard TS, et al. Characterization of the infectionresponsive bovine lactoferrin promoter. Gene. 2005;353:107-117.

24. Li Y, Limmon GV, Imani F, et al. Induction of lactoferrin gene expression by innate immune stimuli in mouse mammary epithelial HC-11 cells. Biochimie. 2009;91:58-67

25. Close MJ, Howlett AR, Roskelley $C D$, et al. Lactoferrin expression in mammary epithelial cells is mediated by changes in cell shape and actin cytoskeleton. J Cell Sci. 1997;110:2861-2871.

26. Liao $Y$, Du X, Lönnerdal B. miR-214 regulates lactoferrin expression and proapoptotic function in mammary epithelial cells. J Nutr. 2010;140:1552-1556.

27. Kabat GC, O'Leary ES, Gammon MD, et al. Estrogen metabolism and breast cancer. Epidemiology. 2006;17:80-88.

28. Muti $P$, Bradlow HL, Micheli A, et al. Estrogen metabolism and risk of breast cancer: a prospective study of the 2:16alpha-hydroxyestrone ratio in premenopausal and postmenopausal women. Epidemiology. 2000;11:635-640.

29. de Martel C, Ferlay J, Franceschi S, et al. Global burden of cancers attributable to infections in 2008: a review and synthetic analysis. Lancet Oncol. 2012;13:607615.

30. Liang R, Lau GK, Kwong YL. Chemotherapy and bone marrow transplantation for cancer patients who are also chronic hepatitis $B$ carriers: a review of the problem. J Clin Oncol. 1999;17:394-398.

31. Furmanski $\mathrm{P}$, Li ZP, Fortuna $M B$, et al. Multiple molecular forms of human lactoferrin. Identification of a class of lactoferrins that possess ribonuclease activity and lack iron-binding capacity. J Exp Med. 1989;170:415-429.

32. Teng C, Gladwell W, Raphiou I, et al. Methylation and expression of the lactoferrin gene in human tissues and cancer cells. Biometals. 2004;17:317-323.

33. Panella TJ, Liu YH, Huang AT, et al. Polymorphism and altered methylation of the lactoferrin gene in normal leukocytes, leukemic cells, and breast cancer. Cancer Res. 1991;51:3037-3043.

34. Liu LH, Gladwell W, Teng CT. Detection of exon polymorphisms in the human lactoferrin gene. Biochem Cell Biol. 2002;80:17-22.
35. Luczak MW, Jagodziński PP. The role of DNA methylation in cancer development Adv Cancer Res. 1990;54:1-23.

36. World Health Organization. GLOBOCAN 2012: estimated cancer incidence, mortality and prevalence worldwide in 2012. Lyon, France: International Agency for Research on Cancer. 2014; Available at: http://globocan.iarc.fr/Pages/ fact_sheets_cancer.aspx. Accessed August 8, 2014.

37. Poullis A, Foster R, Shetty A, et al. Bowel inflammation as measured by fecal calprotectin: a link between lifestyle factors and colorectal cancer risk. Cance Epidemiol Biomarkers Prev. 2004;13:279-284.

38. Corpet DE, Pierre F. Point: from animal models to prevention of colon cancer. Systematic review of chemoprevention in min mice and choice of the model system. Cancer Epidemiol Biomarkers Prev. 2003;12:391-400.

39. Tanaka $\mathrm{T}$, Kawabata $\mathrm{K}$, Kohno $\mathrm{H}$, et al. Chemopreventive effect of bovine lactoferrin on 4-nitroquinoline 1-oxide-induced tongue carcinogenesis in male F344 rats. Jpn J Cancer Res. 2000;91:25-33.

40. Norrby K, Mattsby-Baltzer I, Innocenti M, et al. Orally administered bovine lactoferrin systemically inhibits VEGF(165)-mediated angiogenesis in the rat. Int J Cancer. 2001;91:236-240.

41. Chandra Mohan KV, Kumaraguruparan R, Prathiba D, et al. Modulation of xenobiotic-metabolizing enzymes and redox status during chemoprevention of hamster buccal carcinogenesis by bovine lactoferrin. Nutrition. 2006;22:940946.

42. Ushida Y, Sekine K, Kuhara T, et al. Possible chemopreventive effects of bovine lactoferrin on esophagus and lung carcinogenesis in the rat. Jpn J Cancer Res. 1999;90:262-267.

43. Teraguchi $\mathrm{S}$, Wakabayashi $\mathrm{H}$, Kuwata $\mathrm{H}$, et al. Protection against infections by ora lactoferrin: evaluation in animal models. Biometals. 2004;17:231-234

44. Suzuki YA, Shin K, Lönnerdal B. Molecular cloning and functional expression of a human intestinal lactoferrin receptor. Biochemistry. 2001;40:15771-15779.

45. Yang $\mathrm{N}$, Lejon T, Rekdal $\mathrm{O}$. Antitumour activity and specificity as a function of substitutions in the lipophilic sector of helical lactoferrin-derived peptide. J Pept Sci. 2003;9:300-311.

46. Fillebeen C, Descamps L, Dehouck MP, et al. Receptor-mediated transcytosis of lactoferrin through the blood-brain barrier. J Biol Chem. 1999;274:7011-7017.

47. Eliassen LT, Berge G, Leknessund A, et al. The antimicrobial peptide, lactoferricin $B$, is cytotoxic to neuroblastoma cells in vitro and inhibits xenograft growth in vivo. Int J Cancer. 2006;119:493-500.

48. Yamada $Y$, Sato R, Kobayashi S, et al. The antiproliferative effect of bovine lactoferrin on canine mammary gland tumor cells. J Vet Med Sci. 2008;70:443448

49. Wolf JS, Li G, Varadhachary A, et al. Oral lactoferrin results in T cell-dependent tumor inhibition of head and neck squamous cell carcinoma in vivo. Clin Cance Res. 2007;13:1601-1610.

50. Xu XX, Jiang HR, Li HB, et al. Apoptosis of stomach cancer cell SGC-7901 and regulation of Akt signaling way induced by bovine lactoferrin. J Dairy Sci. 2010;93:2344-2350.

51. Lin TY, Chiou SH, Chen M, et al. Human lactoferrin exerts bi-directional actions on PC12 cell survival via ERK1/2 pathway. Biochem Biophys Res Commun. 2005;337:330-336.

52. Lee SH, Park SW, Pyo CW, et al. Requirement of the JNK-associated BCl-2 pathway for human lactoferrin-induced apoptosis in the Jurkat leukemia $T$ cell line. Biochimie. 2009;91:102-108.

53. Fischer R, Debbabi H, Dubarry M, et al. Regulation of physiological and pathological Th1 and Th2 responses by lactoferrin. Biochem Cell Biol. 2006;84:303311.

54. ligo M, Shimamura M, Matsuda E, et al. Orally administered bovine lactoferrin induces caspase- 1 and interleukin-18 in the mouse intestinal mucosa: a possible explanation for inhibition of carcinogenesis and metastasis. Cytokine. 2004;25:36-44.

55. ligo $\mathrm{M}$, Alexander $\mathrm{DB}$, Long $\mathrm{N}$, et al. Anticarcinogenesis pathways activated by bovine lactoferrin in the murine small intestine. Biochimie. 2009;91:86-101.

56. Kuhara $\mathrm{T}$, ligo $\mathrm{M}$, Itoh $\mathrm{T}$, et al. Orally administered lactoferrin exerts an antimetastatic effect and enhances production of IL-18 in the intestinal epithelium. Nutr Cancer. 2000;38:192-199.

57. Rochard $\mathrm{E}$, Legrand $\mathrm{D}$, Lecocq $\mathrm{M}$, et al. Characterization of lactotransferrin receptor in epithelial cell lines from non-malignant human breast, benign mastopathies and breast carcinomas. Anticancer Res. 1992;12:2047-2051.

58. Bi BY, Liu JL, Legrand D, et al. Internalization of human lactotransferrin by the Jurkat human lymphoblastic T-cell line. Eur J Cell Biol. 1996;69:288-296.

59. Duchardt $F$, Ruttekolk IR, Verdurmen WP, et al. A cell-penetrating peptide derived from human lactoferrin with conformation-dependent uptake efficiency. J Biol Chem. 2009;284:36099-36108.

60. Damiens E, Mazurier J, el Yazidi I, et al. Effects of human lactoferrin on NK cell cytotoxicity against haematopoietic and epithelial tumour cells. Biochim Biophys Acta. 1998;1402:277-287.

61. Ji ZS, Mahley RW. Lactoferrin binding to heparan sulfate proteoglycans and the LDL receptor-related protein. Further evidence supporting the importance of direct binding of remnant lipoproteins to HSPG. Arterioscler Thromb. $1994 ; 14: 2025-2031$ 
62. Liu Y, Han F, Xie Y, et al. Comparative antimicrobial activity and mechanism of action of bovine lactoferricin-derived synthetic peptides. Biometals. 2011;24:1069-1078.

63. Yang N, Stensen W, Svendsen JS, et al. Enhanced antitumor activity and selectivity of lactoferrin-derived peptides. J Pept Res. 2002;60:187-197.

64. Jin $Y$, Hammer J, Pate $M$, et al. Antimicrobial activities and structures of two linear cationic peptide families with various amphipathic $\beta$-sheet and $\alpha$-helical potentials. Antimicrob Agents Chemother. 2005;49:4957-4964.

65. Ellerby HM, Arap W, Ellerby LM, et al. Anti-cancer activity of targeted proapoptotic peptides. Nat Med. 1999;5:1032-1038.

66. Szachowicz-Petelska B, Dobrzynska I, Sulkowski S, et al. Characterization of the cell membrane during cancer transformation. J Environ Biol. 2010;31:845850 .

67. Meng X, Riordan NH, Riordan HD, et al. Cell membrane fatty acid composition differs between normal and malignant cell lines. P R Health Sci J. 2004;23:103106.

68. Fujita K, Matsuda E, Sekine K, et al. Lactoferrin enhances Fas expression and apoptosis in the colon mucosa of azoxymethane-treated rats. Carcinogenesis. 2004;25:1961-1966.

69. Testa JR, Tsichlis PN. AKT signaling in normal and malignant cells. Oncogene. 2005;24:7391-7393.

70. Aoki H, Kang PM, Hampe J, et al. Direct activation of mitochondrial apoptosis machinery by c-Jun N-terminal kinase in adult cardiac myocytes. J Biol Chem. 2002;277:10244-10250.

71. Kaushic C, Frauendorf E, Rossoll RM, et al. Influence of the estrous cycle on the presence and distribution of immune cells in the rat reproductive tract. Am J Reprod Immunol. 1998;39:209-216.

72. June $\mathrm{CH}$. Adoptive $\mathrm{T}$ cell therapy for cancer in the clinic. J Clin Invest 2007;117:1466-1476.

73. Artym J, Zimecki M, Paprocka M, et al. Orally administered lactoferrin restores humoral immune response in immunocompromised mice. Immunol Lett. 2003;89:9-15.

74. Wang WP, ligo M, Sato J, et al. Activation of intestinal mucosal immunity in tumor-bearing mice by lactoferrin. Jpn J Cancer Res. 2000;91:1022-1027.

75. Cao R, Farnebo J, Kurimoto $M$, et al. Interleukin-18 acts as an angiogenesis and tumor suppressor. FASEB J. 1999;13:2195-2202.

76. Okamoto I, Kohno K, Tanimoto T, et al. Development of CD8+ effector T cells is differentially regulated by IL-18 and IL-12. J Immunol. 1999;162:32023211.
77. Kanwar JR, Palmano KP, Sun X, et al. "Iron-saturated" lactoferrin is a poten natural adjuvant for augmenting cancer chemotherapy. Immunol Cell Biol. 2008:86:277-288.

78. Tung YT, Chen HL, Yen CC, et al. Bovine lactoferrin inhibits lung cancer growth through suppression of both inflammation and expression of vascular endothelial growth factor. J Dairy Sci. 2013;96:2095-2106.

79. Beard JL, Dawson H, Piñero DJ. Iron metabolism: a comprehensive review. Nutr Rev. 1996:54:295-317.

80. Valko M, Rhodes CJ, Moncol J, et al. Free radicals, metals and antioxidants in oxidative stress-induced cancer. Chem Biol Interact. 2006;160:1-40.

81. Richardson DR, Kalinowski DS, Lau S, et al. Cancer cell iron metabolism and the development of potent iron chelators as anti-tumour agents. Biochim Biophys Acta. 2009;1790:702-717.

82. Massodi I, Thomas E, Raucher D. Application of thermally responsive elastin-like polypeptide fused to a lactoferrin-derived peptide for treatment of pancreatic cancer. Molecules. 2009;14:1999-2015.

83. Roseanu A, Florian PE, Moisei M, et al. Liposomalization of lactoferrin enhanced its anti-tumoral effects on melanoma cells. Biometals. 2010;23:485-492.

84. McKeown ST, Lundy FT, Nelson J, et al. The cytotoxic effects of human neutrophi peptide-1 (HNP1) and lactoferrin on oral squamous cell carcinoma (OSCC) in vitro. Oral Oncol. 2006;42:685-690.

85. Wang J, Li Q, Ou Y, et al. Inhibition of tumor growth by recombinant adenovirus containing human lactoferrin through inducing tumor cell apoptosis in mice bearing EMT6 breast cancer. Arch Pharm Res. 2011;34:987-995.

86. Wang J, Li Q, Ou Y, et al. Recombination adenovirus-mediated human lactoferrin CDNA inhibits the growth of human MCF-7 breast cancer cells. J Pharm Pharmacol. 2012;64:457-463.

87. Chen $\mathrm{H}$, Qin $\mathrm{Y}$, Zhang $\mathrm{Q}$, et al. Lactoferrin modified doxorubicin-loaded procationic liposomes for the treatment of gliomas. Eur J Pharm Sci. 2011;44:164-173.

88. Macciò A, Madeddu C, Gramignano G, et al. Efficacy and safety of oral lactoferrin supplementation in combination with rHuEPO-beta for the treatment of anemia in advanced cancer patients undergoing chemotherapy: open-label, randomized controlled study. Oncologist. 2010;15:894-902.

89. Beljaars L, Bakker HI, van der Strate BW, et al. The antiviral protein human lactoferrin is distributed in the body to cytomegalovirus (CMV) infection-prone cells and tissues. Pharm Res. 2002;19:54-62.

90. Malam Y, Loizidou M, Seifalian AM. Liposomes and nanoparticles: nanosized vehicles for drug delivery in cancer. Trends Pharmacol Sci. 2009;30:592-599. 